\title{
Chapter 3 \\ Public Perceptions of Science and Technology \\ in Europe, China and India
}

\author{
Virgil Rerimassie, Ma Ying, Krishna Ravi Srinivas and Miltos Ladikas
}

\subsection{Introduction}

Science and technology are held in high esteem by important Chinese, European and Indian political leaders.

Science and technology constitute a primary productive force .... The future of agriculture will eventually lie in bioengineering and other highly advanced technologies. So we must recognize the full importance of science and technology (Deng 1993).

These words of Deng Xiaoping, leader of the People's Republic of China from 1982 to 1987 , turned out to be the cornerstone of the Chinese science and technology policy that followed. Similarly, Jawaharlal Nehru, the first prime minister of

\footnotetext{
V. Rerimassie $(\bowtie)$

Rathenau Instituut, P.O. Box 95366, 2509 CJ The Hague, The Netherlands

e-mail: v.rerimassie@rathenau.nl

M. Ying

Chinese Academy of Science and Technology for Development (CASTED),

8 Yuyuantan South Road, Haidian District 100038, Beijing, China

e-mail: may@casted.org.cn

K.R. Srinivas

RIS Research and Information System for Developing Countries,

Zone IV-B, Fourth Floor, India Habitat Center, Lodhi Road,

New Delhi 110003, India

e-mail: ravisrinivas@ ris.org.in; krsriniv@gmail.com

M. Ladikas

Centre for Professional Ethics, School of Health,

University of Central Lancashire, Brook 317, Preston PR1 2HE, UK

e-mail:mladikas@uclan.ac.uk
} 
post-Independent India gave importance to development of S\&T and emphasized its role in national development and in finding solutions to hunger and poverty. $\mathrm{He}$ advocated inculcation of scientific temper.

Also when we turn to the European Union (EU), we find high hopes for science and technology among political leaders. For instance, in a speech about the EU funding scheme Horizon 2020, Máire Geoghegan-Quinn, the European Commissioner for Research, Innovation and Science, noted that 'research and innovation are the best tools at our disposal to renew our economy and tackle a long list of other major challenges that we face' (Geoghegan-Quinn 2012).

Indeed, science and technology are highly valued by important political leaders of the three regions. But how are they perceived by the citizens those leaders represent? For history has shown that the public reception of science and technology exerts a crucial influence on the development of science and technology.

This chapter provides a brief comparative analysis of public perceptions of science and technology in Europe, China and India. Compiling such an analysis is not an easy task, however. First of all, while research on public perceptions of science and technology has been common practice in Europe and China, this is not the case in India. In addition, the comparability of the available information is limited, since the surveys differ in scope and the questions asked vary widely. Moreover, one has to be aware of the multitude of different cultures and subcultures, belief systems and traditions found in each region, which are manifestly hard to differentiate and analyse in a fair and just manner.

We therefore do not claim to have conducted a comprehensive, detailed analysis, but aim merely to provide an impression of public perceptions of science and technology in the three regions that can serve as a backdrop for the other chapters of this book. Discussions of the case studies of genetically modified foods, nanotechnology and synthetic biology are certainly informed by more general perceptions of science and technology.

To gauge public perceptions of science and technology in the EU, we draw on Eurobarometer, the main series of polling surveys by the European Commission, run regularly in all EU member states. Our primary source of information is the Special Eurobarometer 340 (European Commission 2010). ${ }^{1}$ For China, the national survey of public scientific literacy (Ren et al. 2010, 2011), provides the most comprehensive and systematic statistics concerning public perceptions of science and technology. Since the 1990s, the China Association for Science and Technology has conducted the national survey approximately every 2 years. Information on public perceptions in India is, however, far more scarce: the most relevant and recent study is the India Science Report (NCAER 2005), commissioned by the Indian National Science Academy from the National Council of Applied Economic Research, which included a questionnaire on science and technology perceptions. A key objective was to understand public attitudes to science

\footnotetext{
${ }^{1}$ Occasionally this chapter refers to earlier editions, or to the more recent 2014 report (European Commission 2014). We focus mainly on the 2010 survey, however, because it is the most directly comparable to the surveys of the other regions.
} 
through a primary survey. The sample size was limited, and there have been no subsequent studies based on this questionnaire. Yet it provides a glimpse of Indian public perceptions of science and technology, and moreover contains some directly comparable survey questions.

The following section discusses and compares the key themes of interest in and basic knowledge of science and technology, the image of science and technology and scientists, tensions between science and faith, and the weighing up of the benefits and potential risks of science and technology.

\subsection{Interest and Knowledge Regarding Science and Technology}

How much interest do citizens have in science and technology? All three surveys compared interest in science and technology with interest in other issues.

According to the Eurobarometer report (European Commission 2010), EU citizens consider themselves very, or at least moderately, interested in and informed about issues of everyday life, such as politics, environmental problems and culture and the arts. Regarding new scientific discoveries and technological developments, $79 \%$ of EU citizens are interested (30\% very interested, $49 \%$ moderately so), whereas $20 \%$ are not interested at all. Interest in environmental problems and new medical discoveries is higher, but interest in science and technology is higher than interest in politics, culture, the arts, and sports news. If we compare this with the level of interest in science and technology according to the earlier Eurobarometer surveys of 1993 and 2005, we can conclude that interest in science and technology has been fairly high over the years. ${ }^{2}$ In 2005 about $76 \%$ indicated an interest in science and technology (European Commission 2005), and in 1992 the figure was $82 \%$ (European Commission 1993). ${ }^{3}$ In Special Eurobarometer 401 (European Commission 2014), interest in science and technology was also gauged, but without reference to other issues (politics, culture and arts, sports news etc.). In the 2014 survey, $53 \%$ indicated their interest in scientific and technological developments, compared with $46 \%$ who were not interested. While the question is formulated differently from its equivalent in the 2010 Eurobarometer, the lower numbers in 2014 are certainly notable.

\footnotetext{
${ }^{2}$ Regarding comparisons over time, it is important to keep in mind that the composition of the European Union has changed considerably. The survey work for the Eurobarometer report Europeans, Science and Technology (European Commission 1993), was conducted in 1992, when the European Community, as it was then, consisted of only 12 member states. There were 15 member states in 2001 and 25 in 2005.

${ }^{3}$ In 2001 just $45 \%$ of respondents indicated an interest in science and technology, but this survey posed the question and possible answers differently. The survey question read: 'Are you rather interested or not very interested in each of the following subjects?'. The possible answers were 'rather interested', 'not very interested' and 'do not know'. The level of interest registered in other subjects was lower too, due to the different way the statement was posed (European Commission 2001).
} 
Regarding active interest in science and technology, Eurobarometer 2010 shows that European citizens are actually not very active in science and technology issues. They are most active in donating money to fundraising campaigns for medical research $(39 \%)$, as well as the $13 \%$ of respondents who claim that they sign petitions or take part in street demonstrations on science and technology matters such as nuclear power and biotechnology (European Commission 2010). Overall, we find that EU citizens are indeed interested in science and technology, but this does not translate into an active involvement in science and technology issues.

Like the Eurobarometer survey, the Chinese scientific literacy survey in 2010 included questions on the public's interest in various news topics. Of the 11 topics covered, four clearly related to science and technology: new scientific discoveries, new medical discoveries, suitable production technologies, inventions and new technologies. Generally speaking, developments in science and technology were not the area that attracted the greatest interest. The public was most interested in culture and education (79\%), public security (77\%) and economic development (76\%), followed by agricultural development (73\%), and resources and energy saving $(72 \%)$. The four topics relating to science and technology ranked from sixth to ninth place-new scientific discoveries (72\%), new medical discoveries (71\%), inventions and new technologies (68\%), and suitable production technologies (64\%) -with sports and entertainment and international and foreign policy at the bottom (Ren et al. 2011). Among the four news topics related to science and technology, public interest was highest in new scientific discoveries and new medical discoveries.

Although fewer Chinese respondents expressed an interest in these topics than Europeans, the proportion of the Chinese public explicitly expressing 'no interest' was also lower than that of the EU: only about $7 \%$ said they were 'not interested' in the four topics, while the figure for Europe was more than $17 \%$. Apart from the options 'interested' and 'not interested', a considerable proportion of the Chinese public chose the option 'do not care' or 'do not know': in fact, for all four topics, more than $20 \%$ chose one of these options (Ren et al. 2011), whereas in Europe only $1 \%$ of respondents selected 'do not know'. This shows not only that the Chinese public, compared with the European public, has a lower explicit interest in science and technology, but also, and more significantly, that a higher proportion of the public does not have a clear attitude on the issue.

Turning to India, we find that according to the India Science Report (NCAER 2005), high levels of illiteracy and low levels of income have not prevented Indians from having very high interest in a wide range of social issues as well as a reasonably good knowledge of scientific and other events. Indians profess to be most interested in issues of poverty ( $77 \%)$, followed by those concerning old people (75\%), women (74\%), local schools (71\%) and agriculture (71\%). Only $47 \%$ of those surveyed were interested in economic issues other than employment (in which $66 \%$ were interested). In comparison, interest in science and technology was actually rather low: only $30 \%$ indicated interest, while $40 \%$ said they were not interested. Also striking was that $30 \%$ of the respondents claimed not to have an opinion on this subject matter (NCAER 2005: 45, 46). 
While it is difficult to compare the results from the European, Chinese and Indian surveys directly, we can make some cautious observations. First, notwithstanding a stronger interest in other topics, citizens in the EU and in China do appear to have considerable interest in developments of science and technology: the survey results indicate that more than two thirds expressed an interest in such developments. Indian citizens, however, have a significantly lower degree of interest in science and technology, according to the India Science Report. They are certainly less outspoken on science and technology developments, with almost a third indicating that they had no opinion on whether they had an interest or not.

Level of interest is an important parameter for public engagement with science and technology, as is the level of knowledge of the subject. In all three regions, efforts were made to gauge the level of basic scientific knowledge. In order to do so, the respective surveys incorporated comparable quiz sections, in which true-orfalse questions were asked, testing basic knowledge on, for example, astronomy, biology and physics. These included 'the oxygen we breathe comes from plants', and 'the centre of the Earth is very hot'.

According to the 2005 Eurobarometer (the last edition to address the basic scientific knowledge of European citizens), respondents had a good knowledge of scientific topics. On average, two thirds of the given answers were correct (European Commission 2005). In the India Science Report, almost half of the survey participants gave the correct answers to a similar set of questions: in other words, although illiteracy is widespread in India, a significant percentage of the survey participants had a good basic knowledge of science (NCAER 2005: 50). Last, turning to the most recent Chinese survey of 2011, we find that about $40 \%$ of respondents gave the correct answer (Ren et al. 2011). We may conclude that the basic scientific knowledge in the European Union is high in comparison with India and China.

\subsection{The Image of Science and Technology}

The level of interest in science and technology is important, but it does not tell us anything about the image of science and technology - that is, whether it is viewed in a positive or negative light. The available Chinese, European and Indian surveys include questions that tell us something about the image of science and technology among citizens in the three regions. We will look at responses to the following statements:

- Science and technology can sort out any problem.

- Science and technology will ensure that the planet does not run out of resources.

- Science and technology will create more opportunities for future generations.

- Science and technology will make our lives healthier, easier and more comfortable.

It should be noted that not all of the surveys address all of these issues. While the last is addressed in all three regions, the first three are dealt with in the European and Chinese surveys only. 


\subsubsection{Science and Technology Can Sort Out Any Problem}

First, EU citizens do not believe that science and technology can sort out any problem: 'Only $22 \%$ at the EU27 average indicate that they agree that science and technology can sort out any problem, while a clear majority of $57 \%$ shows disagreement to this statement' (European Commission 2010: 39). This does not significantly deviate from the Eurobarometer report of 2005 (European Commission 2005: 53), but a comparison with the 2001 survey indicates that EU citizens have actually grown more optimistic about science and technology: in that year, $16.5 \%$ agreed and $73 \%$ disagreed with the statement (European Commission 2001: 29).

Interestingly, in the 2005 Chinese survey, this statement drew agreement from $22 \%$ of respondents, significantly fewer than the $39 \%$ of 2003 (Ren et al. 2010). Since the Chinese and EU surveys were conducted in different years, it is not possible to compare the results directly, but one can note the different trends: while the Chinese public is becoming less optimistic, the EU survey points in the opposite direction.

\subsubsection{Science and Technology Will Ensure that the Planet Does Not Run Out of Resources}

Nanotechnologies and synthetic biology are expected to make an important contribution to more sustainable 'bio-based' production strategies. Just as they have little faith in the potential of science and technology to sort out any problem, EU citizens do not regard it as likely that the advances of science and technology will prevent the exhaustion of Earth's natural resources. Only $21 \%$ believe that science and technology will render the Earth's natural resources inexhaustible (European Commission 2010). Comparison with the support level of $23 \%$ in 2005 suggests that EU citizens have grown slightly more sceptical on this subject (European Commission 2005: 53). In 2001, however, the rate of support was just as high as in the most recent survey (European Commission 2001: 29).

Turning to China, we actually find slightly more optimism on this issue than in the EU, with $28 \%$ of the public endorsing the statement in 2010 (Ren et al. 2011) (Fig. 3.1).

\subsubsection{Science and Technology Will Create More Opportunities for Future Generations}

In the EU this statement is certainly supported: in the most recent Eurobarometer three-quarters of respondents agreed and just $7 \%$ disagreed (European Commission 2014). Earlier editions of the survey also reflect widespread agreement: the 2014 and 2010 results are almost identical. This is a slight decrease 
Fig. 3.1 Science and technology will ensure that the earth does not run out of resources

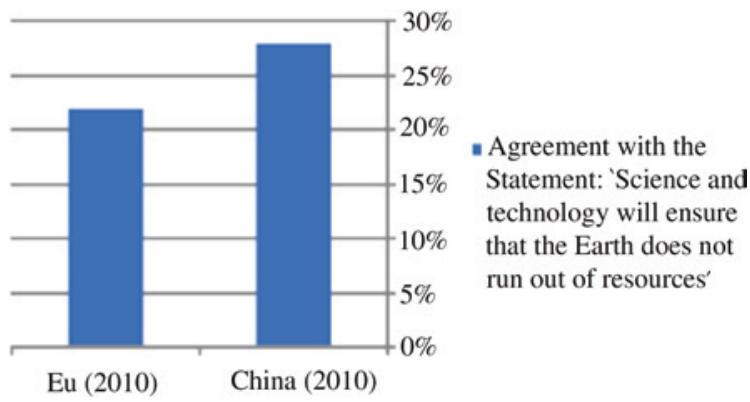

Fig. 3.2 Science and technology will provide more opportunities for future generations

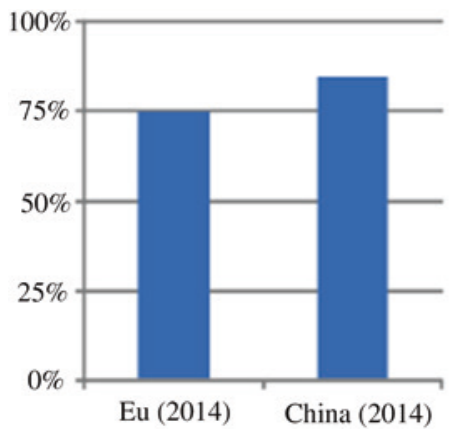

$=$ Agreement to statement 'Science and technology will provide more opportunities for future generations'

on 2005, when support was at $77 \%$. In 2001 it was about $72 \%$ (European Commission 2001: 30). All of these numbers are considerably more favourable than the response in 1992, when $63 \%$ believed science and technology would create more opportunities for future generations (European Commission 1993: 70).

Meanwhile, asked in 2010 whether science and technology would provide more development opportunities for future generations, $85 \%$ of Chinese respondents said yes. Earlier Chinese surveys indicate that support from the Chinese public has always been slightly higher than that of EU citizens (Ren et al. 2011).

As Fig. 3.2 shows, both Chinese and EU public are quite optimistic about science and technology enabling more opportunities or future generations, but there is even more optimism in China than in the EU.

\subsubsection{Science and Technology Will Make Our Lives Healthier, Easier and More Comfortable}

This matter is addressed in all three regional surveys. The Eurobarometer 2010 demonstrates widespread agreement with the statement (including strong agreement) among EU member states, with two thirds (66\%) of respondents agreeing. This level of support was similar in the 2014 Eurobarometer survey, but had, in fact, been even higher in earlier surveys. In 2005, $78 \%$ of the respondents 
Fig. 3.3 Science and technology will make our lives healthier, easier and more comfortable

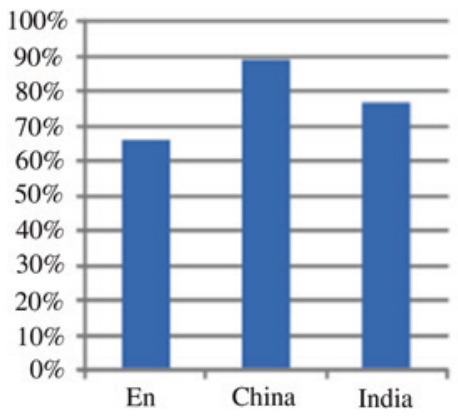

- Agreement to the statement 'Science and technology will make our lives healthier, easier and more comfortable'

subscribed to this statement (European Commission 2005: 53), and in 2001 support was at about $71 \%$ (European Commission 2001: 29). ${ }^{4}$

If citizens of the EU are quite optimistic about science and technology, the Chinese public is even more positive: no less than $89 \%$ of Chinese respondents agreed with this statement in the 2010 survey. As for trends, as many as $94 \%$ of Chinese citizens supported the statement in 2001..$^{5}$ After falling to 83 and $70 \%$ in 2003 and 2005, the support rate climbed back to 76 and $89 \%$ in 2007 and 2010 (Ren et al. 2010, 2011).

The India Science Report indicates that the Indian public is also more optimistic than the EU public, with $77 \%$ of respondents believing that science and technology make lives healthier and more comfortable. The researchers note, however, that there are pronounced differences in perception according to educational and income levels. The report records that just $56 \%$ of illiterate respondents felt that science and technology makes lives healthier, easier and more comfortable, while $98 \%$ of postgraduates felt the same way (NCAER 2005). Yet overall, the balance of opinion was that science and technology benefited the country:

(T)his remains true for all sets of people, ranging from the illiterate to postgraduates and from the bottom-most income quintile to the top-most income quintile. Over three-fourths people in rural India also, for instance, feel that $\mathrm{S} \& \mathrm{~T}$ makes life healthier and easier (against $80 \%$ for urban areas) and $57 \%$ feel that new technology makes work more interesting (68\% for urban areas) (NCAER 2005: 40).

Interestingly, in the first section we observed that the interest of the Indian public in science and technology developments was rather low, and yet they are quite optimistic about the benefits such developments might bring.

Figure 3.3 graphically demonstrates that the public in all three regions are quite certain of the benefits that science and technology may bring, and convinced that science and technology will make their lives healthier, easier and more comfortable. Yet we find remarkable differences in the levels of optimism: in the EU two

\footnotetext{
${ }^{4}$ Strikingly we can observe a steep decline in support for this statement in Germany: from $86 \%$ in 2005 to 57 \% in 2010 and 54 \% in 2014 (European Commission 2005, 2010, 2014).

5 The proposition before 2010 was: 'Generally speaking, the work of scientists has made our life easier and more comfortable.'
} 
thirds agree with the statement, in India about three quarters and in China almost nine out of ten respondents.

\subsection{Fear of Scientists}

We have seen that Chinese and EU citizens do not expect miracles from science and technology: science cannot solve all problems, nor can it ensure that the Earth never runs out of natural resources. They are, however, quite confident that science and technology will enable more opportunities for future generations. We do not know as much about Indian public perceptions of science and technology in this regard as we know of the sentiment in China and the EU. What little information is available, though, points in a similar direction: Chinese, Indian and EU citizens are all quite convinced that science and technology will enable healthier, easier and more comfortable ways of living. Scientific knowledge, however, equals power. How do members of the public view scientists in this regard? Here we can examine the EU and Chinese publics.

More than half of EU citizens (53\%) agree with the statement 'Because of their knowledge, scientists have a power that makes them dangerous,' while $24 \%$ explicitly disagree (European Commission 2010: 42). This fear of scientists has diminished since 2005, however, when an average of $59 \%$ of respondents in the then 25 member states believed that scientists have a power that makes them dangerous (European Commission 2005: 82). In 2001 even more respondents, $63 \%$, agreed with the statement (European Commission 2001: 36).

Few people in China agreed with a comparable statement, namely 'Scientists are scary because they have the knowledge and capability to change the world.' Only $14 \%$ of the Chinese public supported this statement in 2007, and the support rates in 2005 and 2003 were 11 and $15 \%$ respectively (Ren et al. 2010, 2011).

The results of the Chinese survey are therefore set in stark contrast with those of the EU, as more than half of EU citizens see scientists as wielding a power that makes them dangerous. This divergence reveals a major difference between the Chinese and European publics: although the Chinese public is less optimistic on this question than in the past, its cautious attitude is far from materializing into worries and fears for science and technology in general, and its negative attitude and sentiment towards science and technology therefore seem rather limited in scope.

\subsection{Between Science and Faith}

Faith and religion have had a delicate relationship with science and technology throughout history. Therefore both the Eurobarometer survey and the Chinese national survey of public scientific literacy test the proposition: we depend too much on science and not enough on faith. 
The Eurobarometer 2014 report points to a public divide on this statement. On average $39 \%$ of EU citizens agree and $32 \%$ disagree, which is not very different from the 2010 survey. In addition, the reports show that opinions differ greatly between countries, with respondents in the eastern and Mediterranean countries more inclined to agree (European Commission 2010, 2014). On a related subject, most people (58\%) agree that the pace of developments in science and technology makes our ways of life change too fast.

In contrast the Chinese survey conducted in 2007 showed that only $16 \%$ of respondents supported this argument. Moreover, support from the Chinese public shows a steady declining trend: while $23 \%$ of respondents said yes to the question in 2001, the support rate was only $18 \%$ in 2005 and as low as $16 \%$ in 2007 . These results show that the Chinese public is clearly not as worried about the issue as the EU public. Based hereon, we may wonder whether the Chinese public is disinclined to consider and judge issues arising from science and technology developments from the perspective of the dominant belief system, than the European public.

\subsection{Balancing Risks and Benefits}

Nanotechnology, genetically modified crops and synthetic biology all hold the promise of major benefits for society. However, they also have in common that they raise potential risks. In this section we discuss how EU, Chinese and Indian citizens perceive the benefits that science and technology may bring and the risks that these entail.

'Close to half of Europeans, $46 \%$ of respondents at the EU27 level, agree that the benefits of science are greater than any harmful effects it may have' (European Commission 2010: 74). However, $20 \%$ disagree. The level of agreement has declined since earlier surveys (European Commission 1993: 74, 2001: 29, 2005: 75). This raises the issue of what role the precautionary principle should have in coping with science and technology, which was examined through the statement: 'If a new technology poses risks that are uncertain and not yet fully understood, the development of this technology should be stopped even if benefits are expected' (European Commission 2010: 78). Interestingly, the survey demonstrates that almost one in two (49\%) EU citizens agree with this statement, while only $22 \%$ disagree. To make the situation even more complicated, 'while Europeans express the need for risk management, at the same time they do not want to miss out on technological progress. A slim majority of $52 \%$ of respondents at the EU27 level agree that technological progress will be slowed down if risks that are not yet fully understood receive too much importance' (European Commission 2010: 81).

Once again the Chinese public seems more optimistic: almost three-fourths (74\%) of respondents indicated that science and technology could bring good and bad and the good is always more than the bad'. In earlier years, however, the 
support rate was slightly lower, but in any case significantly higher than that of EU citizens on this issue (Ren et al. 2010, 2011).

The issue is not addressed in the India Science Report, but it does ask whether 'technologies will eventually destroy the Earth', which evidently tells us something about the perception among the Indian public of the risks involved in science and technology. The report shows that a striking $39 \%$ agree with this statement, while $25 \%$ disagree. Another $36 \%$ answered 'do not know' (NCAER 2005: 109).

In sum: EU citizens and Indian citizens perceive science and technology very much as a double-edged sword. On the one hand, they are quite confident about its potential benefits and, in case of the EU, do not really want to miss out. On the other hand, they are quite wary of the potential downsides of developments in science and technology. The Chinese public, on the other hand, is optimistic about the benefits and convinced that the potential downsides can be accommodated.

\subsection{Conclusion}

What have we learned from this comparison? It is hard to draw elaborate conclusions in the absence of fully comparable data sets. But while we work towards establishing more comparable surveys, it would be foolish to disregard the obvious opportunity to draw certain cautious conclusions from the material that is available.

Science and technology perceptions are, overall, positive in the three regions. Interest in science and technology varies between the high levels of the EU and China and a somewhat lower level in India. This might be the result of access to science and technology developments in everyday life currently being higher in the EU and China than in India. Interest grows with increased exposure to science and technology. Interest does not, though, necessarily translate into involvement, as we have seen in the case of EU. Actual engagement with science and technology issues relates to proximity to technological developments (e.g. genetically modified food), while the incorporation of engagement into policy-making requires the establishment of official structures that might not exist in every region yet (see Chap. 2).

The overall image of science and technology is positive in all three regions, and this does not appear to be related to one's knowledge level, educational background or income. Rich and poor, literate and illiterate find the promise of science and technology compelling and worth supporting. The fact that most people do not regard science and technology as a panacea for the problems society faces is merely the reflection of a pragmatic attitude on the part of citizens who have experienced financial crises, unemployment or even a lack of basic amenities. It does not translate into lower support for science and technology.

What appears to put the brakes on people's optimism is the awareness that power can serve good as well as bad aims. The power that individuals (i.e. scientists) can wield is problematic for Europeans, while it does not appear to be an 
issue for the Chinese public (yet). This could reflect the instinctive uneasiness that the average European would have with the concentration of power in few hands, something that the average Chinese might perhaps be less worried about.

A related matter is the perception of a precarious balance between faith and science in the minds of the more religious European publics, in contrast to the less religious Chinese public. Here one recalls the uneasy dichotomy between faith and state that developed during the Enlightenment in Europe; in China, however, the dominant belief system has happily incorporated science and technology as a main pillar, thus easing the potential tension between traditional beliefs and new developments. As for India, one can only make an informed guess that the tension between faith on one hand and science and technology on the other is real and strong in the most traditional sectors of society (see Chap. 7).

Finally, perceptions of risks and benefits vary in the three regions, demonstrating that this is a very volatile variable, depending on the focus of the questions (i.e. the specific technology) and the current social context (e.g. recent scandals and media attention). Few people in the three regions see science and technology as without risk, but most retain a guarded optimism about the overall benefits. When it comes to application of the precautionary principle, the EU leads support, naturally influenced by the widespread debates in Europe on genetically modified foods and crops. Increasing support for precaution can certainly be expected in China and India too, once the debates there acquires a focus similar to what we have seen elsewhere (for instance, see Chap. 9).

Overall, the available comparisons provide many valuable insights into state of affairs in the three regions, but they also highlight the need to create fully comparable surveys to facilitate detailed analysis of the parameters affecting science and technology perceptions. Once such surveys have been executed, then our understanding of the three regions will increase substantially, and our aim of promoting meaningful science and technology collaborations among them will be more easily realized.

Open Access This chapter is distributed under the terms of the Creative Commons Attribution Noncommercial License, which permits any noncommercial use, distribution, and reproduction in any medium, provided the original author(s) and source are credited.

\section{References}

Deng X (1993) Deng Xiaoping anthology, vol 3. People's Press, Beijing

European Commission (1993) Europeans, science and technology: public understanding and attitudes. European Commission, Brussels

European Commission (2001) Special Eurobarometer 55.2: Europeans, science and technology. European Commission, Brussels

European Commission (2005) Special Eurobarometer 224: Europeans, science and technology. European Commission, Brussels

European Commission (2010) Special Eurobarometer 340: science and technology. European Commission, Brussels 
European Commission (2014) Special Eurobarometer 401: responsible research and innovation, science and technology. European Commission, Brussels

Geoghegan-Quinn M (2012) Research and innovation in Europe: the role of public-private partnerships in Horizon 2020. In: Conference of the Italian association of technology districts (ADITE), Rome, 3 May 2012

NCAER (2005) India science report: science education, human resources and public attitude towards science and technology. National Council of Applied Economic Research, New Delhi

Ren F et al (2010) Report on scientific literacy of Chinese citizens, vol 1. Popular Science Press, Beijing

Ren F et al (2011) Report on scientific literacy of Chinese citizens, vol 2. Popular Science Press, Beijing 